\title{
Escherichia coli heat-shock proteins IbpA and IbpB affect biofilm formation by influencing the level of extracellular indole
}

\author{
Dorota Kuczyńska-Wiśnik, Ewelina Matuszewska and Ewa Laskowska
}

Correspondence

Ewa Laskowska

lasko@biotech.ug.gda.pl

Received 2 July 2009

Revised 23 September 2009

Accepted 29 September 2009
Department of Biochemistry, Faculty of Biology, University of Gdańsk, Kładki 24, 80-952 Gdańsk, Poland

The development of Escherichia coli biofilm requires the differential expression of various genes implicated in cell signalling, stress responses, motility and the synthesis of structures responsible for cell attachment. The ibpAB operon is among the stress-response genes most induced during growth of the $E$. coli biofilm. In this study we demonstrated, to our knowledge for the first time, that the lack of IbpAB proteins in $E$. coli cells inhibited the formation of biofilm at the air-liquid interface, although it allowed normal planktonic growth. We showed that $i b p A B$ mutant cells experienced endogenous oxidative stress, which might result from a decreased catalase activity. The endogenous oxidative stress in ibpAB cells led to increased expression of tryptophanase, an enzyme which catalyses the synthesis of indole. We demonstrated that the formation of biofilm by the $i b p A B$ mutant was delayed due to the increase in the extracellular concentration of indole, which is known to play the role of a signal molecule, inhibiting biofilm growth.

\section{INTRODUCTION}

In natural environments, rather than existing as single freefloating (planktonic) cells most bacteria live attached to surfaces in complex biofilm communities (Hall-Stoodley et al., 2004; Harrison et al., 2007). Ordered steps of biofilm development can be defined: reversible attachment to a surface, irreversible attachment, maturation, and the dispersion of cells to a planktonic state (Prüß et al., 2006; Wood, 2009). Mature biofilms often form three-dimensional, complex and dynamic structures with the attributes of a multicellular organism (Hall-Stoodley et al., 2004; Harrison et al., 2007). The environmental conditions (e.g. microscale nutrient and oxygen concentrations) are not homogeneous throughout a biofilm; therefore, during adaptation to the local environment, sessile micro-organisms form subpopulations of cells (microcolonies) which express distinct metabolic pathways, stress responses and other specific activities (Stewart \& Franklin, 2008). This physiological heterogeneity in biofilms results from significant changes in gene expression occurring during each step of biofilm development (Prüß et al., 2006). The reprogramming of gene expression is also responsible for differentiation in the phenotype of planktonic and sessile cells. Biofilm cells are often more resistant to various stresses (antibiotics, oxidative stress, heavy metals) than their planktonic counterparts (Fux et al., 2005; Harrison et al., 2007). Therefore, formation of biofilm can be

Abbreviations: DNP, 2,4-dinitrophenol; DNPH, 2,4-dinitrophenylhydrazine; $\mathrm{H}_{2} \mathrm{DCFDA}, 2^{\prime}, 7^{\prime}$-dichlorofluorescein diacetate; PVC, polyvinyl chloride; SOD, superoxide dismutase. regarded as a survival strategy of bacteria in hostile environments. However, biofilms can cause serious problems in medicine and industry. The antibiotic-resistant biofilms of pathogenic bacteria may be responsible for many chronic infections (Fux et al., 2005), while in industry, biofilms often cause fouling and the corrosion of metal surfaces (Harrison et al., 2007).

Although the process of biofilm development has been extensively investigated using several bacterial strains, its molecular mechanism is not fully known. Numerous recent studies concerning Escherichia coli biofilm have been based mainly on whole-transcriptome profiling. These reports indicate that the temperature, type of medium and other growth conditions determine which genes are induced or repressed during biofilm development (Schembri et al., 2003; Beloin et al., 2004; Ren et al., 2004; Domka et al., 2007). Analysis of the temporal development of E. coli K-12 biofilms revealed that differentially expressed genes (biofilm versus biofilm, and biofilm versus planktonic cells) belong to several functional categories and are implicated in stress response, quorum sensing, motility, production of fimbriae, and sulfur and tryptophan metabolism and transport (Domka et al., 2007).

Among the stress-response genes overexpressed in biofilm populations, the $i b p A$ and $i b p B$ genes have been identified (Schembri et al., 2003; Ren et al., 2004; Junker et al., 2007). The function of the IbpA and IbpB proteins in biofilm development has not yet been established. The ibpA and $i b p B$ genes form an operon controlled by the $\sigma^{32}$ and $\sigma^{54}$ subunits of RNA polymerase (Kuczyńska-Wisnik et al., 
2001) and are induced under heat- and other stress conditions (Allen et al., 1992; Chuang et al., 1993; Laskowska et al., 2003; Peréz et al., 2007; Nobre et al., 2009). The IbpA and IbpB proteins share about $50 \%$ homology and belong to the ubiquitous family of small heatshock proteins (sHsps). sHsps are characterized by a relatively low molecular mass $(12-30 \mathrm{kDa})$, an oligomeric structure and a conserved C-terminal domain of approximately 100 amino acid residues (Haslbeck et al., 2005; Nakamoto \& Vigh, 2007). This so-called ' $\alpha$-crystallin' domain is homologous to the mammalian eye lens sHsp $(\alpha$-crystallin), which prevents protein aggregation and cataract formation in the lens. IbpA and IbpB were identified as components of inclusion bodies (Allen et al., 1992) and aggregates of heat-denatured proteins (Laskowska et al., 1996). In vitro studies revealed that IbpA and IbpB form complexes with denatured proteins and facilitate their subsequent refolding by the ATP-dependent DnaK-DnaJGrpE chaperone system in cooperation with the $\mathrm{AAA}^{+}$ protein ClpB (Veinger et al., 1998; Mogk et al., 2003a, b; Matuszewska et al., 2005). Both IbpA and IbpB are required in vitro, to efficiently stabilize denatured protein in a folding-competent state; however, they fulfil different roles in this process. IbpA prevents formation of large aggregates of the substrate, but simultaneously inhibits ClpB-DnaKdependent refolding. IbpB interacts with aggregates via IbpA and alleviates the IbpA-mediated inhibitory effect (Ratajczak et al., 2009). Deletion of the $i b p A B$ operon causes increased aggregation of heat-denatured proteins and reduces cell viability under severe heat stress (Kuczyńska-Wisńik et al., 2002). At lower heat-shock temperatures $\left(45^{\circ} \mathrm{C}\right)$ the phenotype of the $i b p A B$ mutation is observed in the triple mutant $i b p A B c l p B$ and in $i b p A B$ cells with downregulated DnaK/DnaJ levels (Mogk et al., 2003a). Recently, we found that IbpA and IbpB participate in the defence against oxidative stress induced by copper. In the presence of oxygen, $i b p A B$ cells exhibit increased sensitivity to copper ions and accumulate elevated amounts of oxidized proteins (Matuszewska et al., 2008).

The results presented in this paper provide new insights into the role of IbpAB during biofilm development. We demonstrate that the lack of IbpAB proteins in E. coli cells results in endogenous oxidative stress and overproduction of extracellular indole, which inhibits the growth of the biofilm at the air-liquid interface.

\section{METHODS}

Bacterial strains, growth conditions and plasmids. E. coli W3110 [F $\mathrm{F}^{-}$lam $\left.r p o S(A m) r p h-1 \operatorname{Inv}(r r n D-r r n E)\right]$ (Colón-González et al., 2004) and its derivatives were used in this study. The W3110 $\triangle i b p A B:: \mathrm{cm}$ mutant was generated by P1 transduction (Sambrook et al., 1989) of W3110 with phage grown on MC4100 $\triangle i b p A B:: \mathrm{cm}$ (Kuczyńska-Wisńik et al., 2002). The ibpAB mutation was verified by Western blotting using anti-IbpAB serum. W3110 $\Delta$ katE731:: kan, W3110 $\Delta$ katG729::kan and W3110 $\Delta$ tnaA::Tn10 were constructed by P1 transduction using E. coli JW1721-1, JW3914-1 and JC12337 (E. coli Genetic Stock Center, Yale University), respectively, as donors.
Both W3110 katE and W3110 katG strains exhibited decreased (by $30-50 \%$, depending on the growth state) catalase activity when compared to W3110.

To grow biofilms, the overnight cultures were diluted $1: 50$ into fresh LB medium supplemented with $0.1 \mathrm{M}$ MOPS $\mathrm{pH} 7.4$ and $0.2 \%$ glucose and incubated for up to $24 \mathrm{~h}$, without shaking, at $30^{\circ} \mathrm{C}$ in $96-$ well polyvinyl chloride (PVC) microtitre plates, using $150 \mu \mathrm{l}$ of culture per well. Plasmid pGab was constructed by cloning between the EcoRI and BamHI sites of plasmid pGB2 (Churchward et al., 1984) a PCR fragment containing the $i b p A B$ operon with its own $\sigma^{32}$ promoter. The PCR fragment was generated with primers $5^{\prime}$ ATGGAATTCACCATAAACTGCA-3' and 5'-TATCACGGATCCGAACGTGC-3', using E. coli chromosomal DNA as the template, and digested with EcoRI and BamHI.

Visualization and quantification of the $E$. coli biofilm. At the indicated time points, bacterial growth was determined by measuring the optical density $\left(\mathrm{OD}_{595}\right)$. After removing the planktonic cells from the PVC wells, the biofilm was stained with $0.1 \%$ crystal violet for 10 min (Colón-González et al., 2004). After rinsing with water, the attached crystal violet was eluted with a mixture of $80 \%$ ethanol and $20 \%$ acetone and quantified spectrophotometrically $\left(A_{595}\right)$ to calculate the relative amount of biofilm $\left(A_{595} / \mathrm{OD}_{595}\right)$.

Enzyme assays. Cells grown in PVC microtitre plates were collected $(1.5 \mathrm{ml})$ at different time points of growth, indicated in the figures, and sedimented. The pellets were washed, resuspended in $300 \mu 10 \mathrm{mM}$ Tris/ $\mathrm{HCl} \mathrm{pH} 7.4$ and disrupted by sonication (Vibra-Cell sonicator, three $5 \mathrm{~s}$ bursts at $20 \%$ power output, with cooling in ice). To determine the catalase activity, the cell extracts were added to $20 \mathrm{mM}$ $\mathrm{H}_{2} \mathrm{O}_{2}$ and the rate of decrease in $A_{240}$ was measured immediately (Jakubowski et al., 2000). Superoxide dismutase (SOD) activity in cell extracts was determined by a colorimetric method using a SOD Assay kit-WST according to the protocol (Fluka Analytical). Tryptophanase activity was assayed according to Choi et al. (2005). A $0.2 \mathrm{ml}$ sample of the cell extract was mixed with $0.2 \mathrm{ml}$ of the reaction mixture $(2.75 \mathrm{mg}$ sodium pyrophosphate, $19.6 \mathrm{mg}$ disodium EDTA dihydrate and $10 \mathrm{mg}$ BSA in $100 \mathrm{ml} 0.05 \mathrm{M}$ potassium phosphate, $\mathrm{pH} 7.5)$ and $0.2 \mathrm{ml}$ $20 \mathrm{mM}$ tryptophan. After $1 \mathrm{~h}$ incubation at $37^{\circ} \mathrm{C}$ the reaction was stopped by adding $2 \mathrm{ml}$ colour reagent solution (14.7 g p-dimethylaminobenzaldehyde, $52 \mathrm{ml} \mathrm{H}_{2} \mathrm{SO}_{4}$ and $948 \mathrm{ml} 95 \%$ ethanol) and the $A_{540}$ was measured. Strain W3110 tnaA:: Tn10 was used as a negative control for this measurement.

Extracellular indole assay. The method of Domka et al. (2006) was used. To measure extracellular indole concentrations in E. coli cultures at different time points of growth (see figures), $2 \mathrm{ml}$ Kovac's reagent (10 g p-dimethylaminobenzaldehyde, $50 \mathrm{ml} \mathrm{HCl}$ and $150 \mathrm{ml}$ amyl alcohol) was mixed for 2 min with $5 \mathrm{ml}$ of medium collected after sedimentation of the bacteria. The reaction mixture was diluted $1: 10$ in $\mathrm{HCl} /$ amyl alcohol solution $(50 \mathrm{ml} \mathrm{HCl}$ and $150 \mathrm{ml}$ amyl alcohol) and the $A_{540}$ was measured. The concentration of indole was calculated based on the calibration curve. Strain W3110 tnaA:: Tn10, which does not produce indole, was used as a negative control.

$\mathbf{2}^{\prime}, \mathbf{7}^{\prime}$-Dichlorofluorescein oxidation. The level of intracellular oxidation was measured in exponential- and stationary-phase cultures using $2^{\prime}, 7^{\prime}$-dichlorofluorescein diacetate $\left(\mathrm{H}_{2} \mathrm{DCFDA}\right)$ according to Jakubowski et al. (2000). Bacteria were sedimented by centrifugation, washed and resuspended in $50 \mathrm{mM}$ sodium phosphate buffer $\mathrm{pH} 7,4$. $5 \mathrm{mM} \mathrm{H} \mathrm{H}_{2}$ DCFDA (dissolved in DMSO) was added to a final concentration of $10 \mu \mathrm{M}$ and samples were incubated at $30{ }^{\circ} \mathrm{C}$. After $30 \mathrm{~min}$, the bacteria were pelleted, washed, suspended in the buffer and disrupted by sonication. The cell extracts were clarified by centrifugation and fluorescence of the supernatant was measured using a Perkin-Elmer LS-5B spectrofluorimeter (excitation, $490 \mathrm{~nm}$; 
emission, $519 \mathrm{~nm}$ ). The emission values were normalized by the protein concentration.

Immunodetection of IbpAB and oxidized proteins. Immunodetection of $\mathrm{Ibp} A B$ in cell extracts was carried out using IbpAB-specific antiserum as primary antibodies (Kuczyńska-Wisńik et al., 2002), with anti-rabbit IgG horseradish peroxidase conjugate (Sigma) and ECL detection reagents (Pierce Biotechnology). The major products of protein oxidation, carbonyl groups, were detected as described by Matuszewska et al. (2008). Briefly, crude protein extracts were derivatized with 2,4-dinitrophenylhydrazine (DNPH) and subjected to SDS-PAGE and Western blotting (Sambrook et al., 1989). The DNPH-derivatized proteins were immunodetected using anti-DNP antibodies (Sigma).

\section{RESULTS}

\section{The ibpAB mutation inhibits formation of $E$. coli W3110 biofilm at the air-liquid interface}

To form biofilm, the W3110 WT and ibpAB strains were grown at $30{ }^{\circ} \mathrm{C}$ in PVC microtitre plates containing LBMOPS medium. The ibpAB mutation did not affect growth (Fig. 1a), but it did influence biofilm formation (Fig. 1b). The wild-type (WT) biofilm appeared after $8 \mathrm{~h}$ of growth and after crystal violet staining was visible as a ring at the air-liquid interface of the cultures (Fig. 1b). The amount of WT air-liquid interface biofilm increased during further incubation up to $24 \mathrm{~h}$. Formation of the $i b p A B$ biofilm was significantly delayed: after $12 \mathrm{~h}$ the amount of $i b p A B$ cells attached to the PVC surface was twofold lower than in the WT cultures. However, the relative amounts of the $i b p A B$ and WT biofilms reached almost the same levels after $24 \mathrm{~h}$ (Fig. 1b) and afterwards decreased gradually at comparable rates (data not shown). The reduction of the WT biofilm during the late stationary phase was in agreement with previously published data (Colón-González et al., 2004). The static cultures cultivated in the microtitre plates probably reach anaerobiosis at the stationary phase. These conditions might be a signal for the detachment of E. coli biofilm.

The level of the IbpAB proteins in the WT cells increased gradually (two- to threefold) during the exponential phase of growth and reached its maximum after $8 \mathrm{~h}$ (Fig. 1c), approximately at the time when the biofilm became visible. Thereafter, the IbpAB level stayed nearly constant and was comparable in planktonic and biofilm cells (Fig. 1c). The 2D-PAGE used for separation of IbpA from IbpB revealed that at $16 \mathrm{~h}$ the IbpA:IbpB molar ratio was $1.5: 1$ in planktonic as well as in biofilm cells (data not shown).

We have previously found that the lack of IbpAB chaperones increases the sensitivity of $E$. coli to oxidative stress induced by copper ions (Matuszewska et al., 2008); therefore we were also interested in whether the presence of $\mathrm{Cu}(\mathrm{II})$ would influence the formation of WT and $i b p A B$ biofilms. We found that the development of the WT biofilm was inhibited in the presence of $\mathrm{Cu}$ (II). After $8 \mathrm{~h}$ the WT biofilm was hardly detectable and its amount was 3.5 -fold lower than that formed in the absence of $\mathrm{Cu}(\mathrm{II})$ (compare Fig. 1b and Fig. 1e). An additional reduction of the biofilm was observed in the $i b p A B$ culture exposed to $\mathrm{Cu}(\mathrm{II})$. In the presence of $\mathrm{Cu}(\mathrm{II})$ the $i b p A B$ biofilm appeared $4 \mathrm{~h}$ later (Fig. 1e) than in the non-treated cultures (Fig. 1b). Moreover, the difference between the $\mathrm{WT}$ and $i b p A B$ biofilms was more pronounced in cultures exposed to $\mathrm{Cu}$ (II) (Fig. 1e) than in the non-treated bacteria (Fig. 1b). It should be noted that since the cultures were incubated without shaking, WT and $i b p A B$ cells were grown in the presence of limited amounts of oxygen. Under these microaerobic conditions, in the presence of $\mathrm{Cu}(\mathrm{II})$, the $i b p A / B$ mutation caused moderate inhibition of E. coli growth (Fig. 1d). The above results indicated that under some stress conditions [Cu(II)-induced stress] bacteria lacking $\mathrm{IbpAB}$ proteins could grow as free-floating cells at rates comparable with WT cells. However, the ability of the $i b p A B$ mutant to form a sessile population biofilm - was seriously impaired under these conditions. Complementation experiments confirmed that the $i b p A B$ mutation was responsible for the observed phenotype. Low-copy-number plasmid pGab, carrying the $i b p A B$ operon, restored the ability of the $i b p A B$ cells to form biofilm at the air-liquid interface (Fig. 1f).

\section{Planktonic and biofilm ibpAB cells contain an increased level of tryptophanase}

To reveal the mechanism responsible for the suppression of $i b p A B$ biofilm formation, we compared the protein profiles of the WT and $i b p A B$ cultures at different stages of biofilm growth. We found that both planktonic and attached ibp $A B$ cells accumulated a $\sim 50 \mathrm{kDa}$ protein during the stationary phase of growth (12-16 h) (Fig. 2a). The cell extracts were resolved by 2D-PAGE (data not shown) to isolate the $50 \mathrm{kDa}$ protein, which was identified by mass spectrometry as tryptophanase (TnaA). It is known that the expression of tnaA is induced at the stationary phase of growth (Lacour \& Landini, 2004), at alkaline $\mathrm{pH}$ (Blankenhorn et al., 1999) and during oxidative stress (Pomposiello et al., 2001; Zheng et al., 2001). TnaA converts tryptophan to indole, ammonia and pyruvate. It has been proposed that indole acts as an extracellular signal which inhibits biofilm formation (Lee et al., 2007). Therefore, we hypothesized that the delayed adhesion of the ibpAB cells to the PVC surfaces might result from the enhanced activity of TnaA and the increased synthesis of indole. Indeed, we found that the activity of TnaA (Fig. 2b) was significantly (1-5-3-fold) higher in the mutant strain throughout the whole course of the experiment, except the late stationary phase $(24 \mathrm{~h})$, when the enzyme activity was comparable in both strains. Enhanced TnaA activity in $i b p A B$ cells might be responsible for the increased concentration of extracellular indole - after $16 \mathrm{~h}$ its level in the $i b p A B$ culture was ninefold higher than in the WT strain (Fig. 2c). Complementation experiments confirmed that the IbpAB proteins influence the level of extracellular indole. $\mathrm{WT}(\mathrm{pGB} 2)$ and $i b p A B(\mathrm{pGab})$ cultures produced comparable amounts of extracellular indole (Fig. 2c). 
(a)

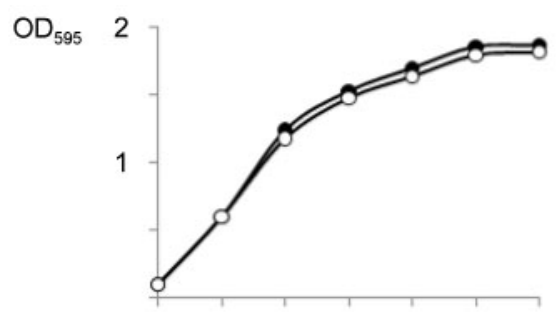

(b)
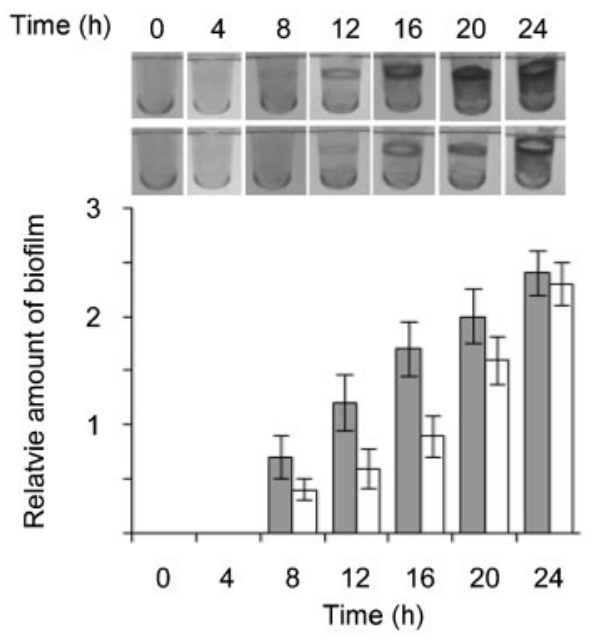

(c)

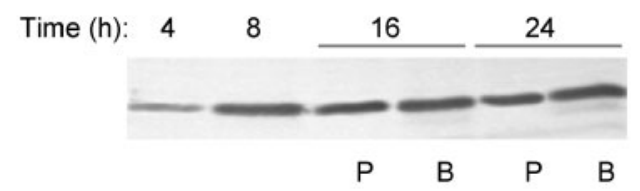

(d)

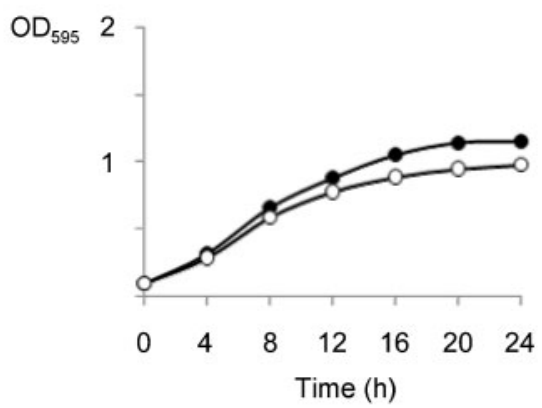

(e)

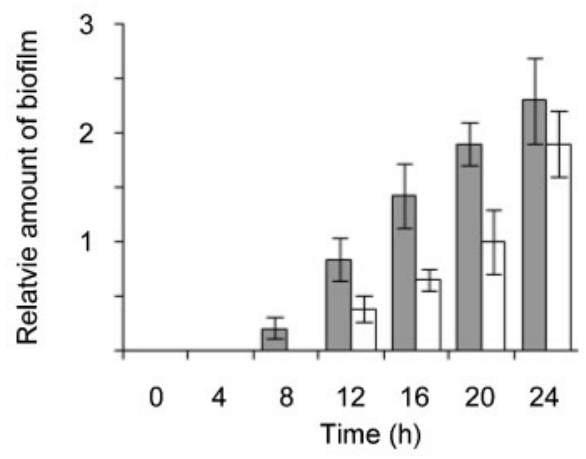

(f)

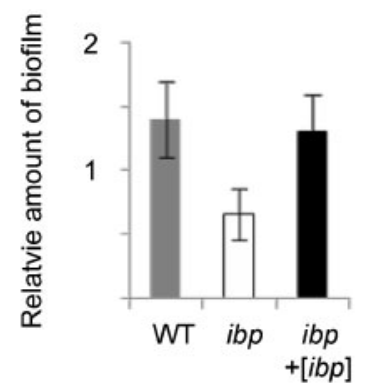

Fig. 1. Lack of IbpAB proteins delays formation of $E$. coli biofilm at the air-liquid interface. $E$. coli WT and ibpAB strains were grown in the absence $(\mathrm{a}-\mathrm{c})$ or presence $(\mathrm{d}, \mathrm{e})$ of $2 \mathrm{mM} \mathrm{CuCl}_{2}$ in $\mathrm{PVC}$ microtitre plates as described in Methods. (a, d) Growth of WT $(\bullet)$ and $i b p A B(\bigcirc)$ cells. (b, e) Biofilm formation. Biofilms were stained with crystal violet at the indicated time points; in (b), for each time point, one representative well of 12 replicate wells is shown (WT, upper row, ibpAB, lower row). Relative amounts of the WT (grey bars) and ibpAB (white bars) biofilms were estimated as described in Methods. Means \pm SD of three independent experiments are shown. In each experiment, 12 wells were averaged to obtain each data point. (c) The level of $\mathrm{IbpAB}$ proteins increases during the exponential phase. IbpAB proteins were detected in whole WT cultures (containing both planktonic and biofilm cells, $4 \mathrm{~h}$ and $8 \mathrm{~h}$ ) and in samples containing planktonic (P) or biofilm (B) cells (16 h and $24 \mathrm{~h})$. Aliquots of samples corresponding to the same amounts of bacteria were separated by SDS-PAGE and immunodetected using anti$\mathrm{IbpAB}$ serum and ECL reagents. The IbpA and IbpB proteins migrated in the polyacrylamide gel as one band and were detected by the serum. (f) Complementation of the ibpAB mutant using the low-copy-number plasmid pGab and empty plasmid pGB2 as a control. E. coli WT(pGB2) (grey bar), ibpAB(pGB2) (white bar) and ibpAB (pGab) (black bar) were grown in the presence of $2 \mathrm{mM} \mathrm{CuCl}_{2}$. Relative amounts of biofilm were determined after $16 \mathrm{~h}$.

It has been reported that temperature affects indole signalling in $E$. coli and indole reduces biofilm formation more significantly at $30{ }^{\circ} \mathrm{C}$ than at $37^{\circ} \mathrm{C}$ (Lee et al., 2008). Therefore, the effect of the $i b p A B$ mutation (delayed biofilm formation) should be suppressed at $37{ }^{\circ} \mathrm{C}$. Indeed, we did not observe any substantial differences in the formation of WT and $i b p A B$ biofilms at $37^{\circ} \mathrm{C}$ (Fig. 3), in spite of the fact that the amount of indole in the $i b p A B$ culture was still some five-to sevenfold higher than in the WT strain (data not shown). Lee et al. (2008) demonstrated that the addition of exogenous indole reduces E. coli biofilm formation in a dose-dependent manner. Under the conditions applied in our studies we obtained a similar result (data not shown). Taken together, these data suggest that the increased concentration of extracellular indole was responsible for the delay of the $i b p A B$ biofilm formation. 
(a)

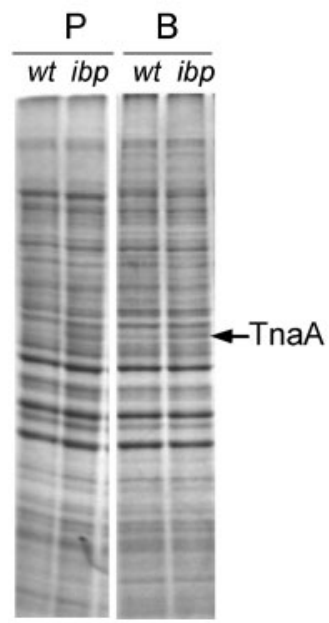

(b)

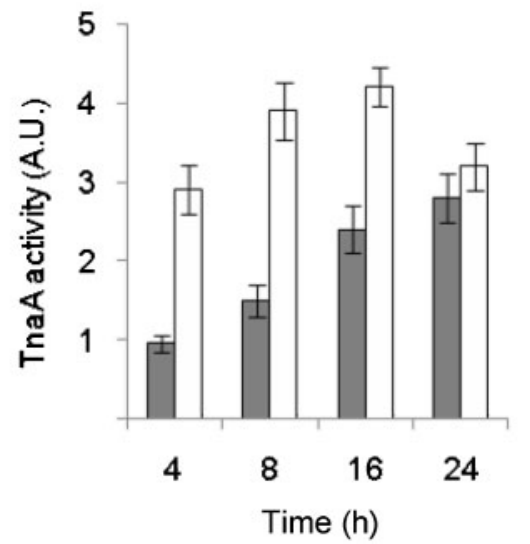

(c)

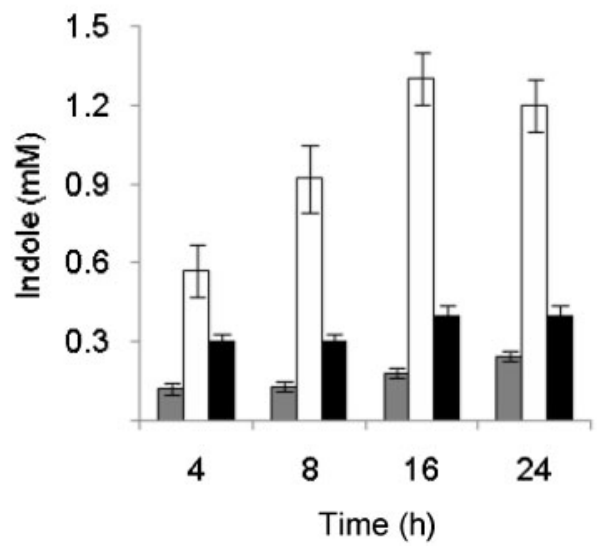

Fig. 2. TnaA activity and extracellular indole concentration are increased in $E$. coli cells lacking lbpAB proteins. $E$. coli WT and ibpAB cells were grown in PVC microtitre plates for $16 \mathrm{~h}$ as described in the legend to Fig. 1. (a) Planktonic (P) and biofilm (B) cells were collected separately and samples containing equal amounts of protein were resolved by SDS-PAGE. The gel was stained with Coomassie brilliant blue. (b) TnaA activity was measured in cell extracts obtained from whole E. coli cultures containing both planktonic and biofilm cells, A.U., arbitrary units. Grey bars, WT; white bars, ibpAB. (c) The extracellular level of indole was determined in the spent medium, after sedimentation of planktonic cells. Grey bars, WT(pGB2); white bars, ibpAB(pGB2); black bars, ibpAB(pGab). TnaA activities and extracellular indole levels were normalized to cell density. Means \pm SD of three independent experiments are shown.

\section{ibpAB cells are exposed to endogenous oxidative stress}

Our next aim was to identify the signal responsible for the increased synthesis of TnaA and extracellular indole in

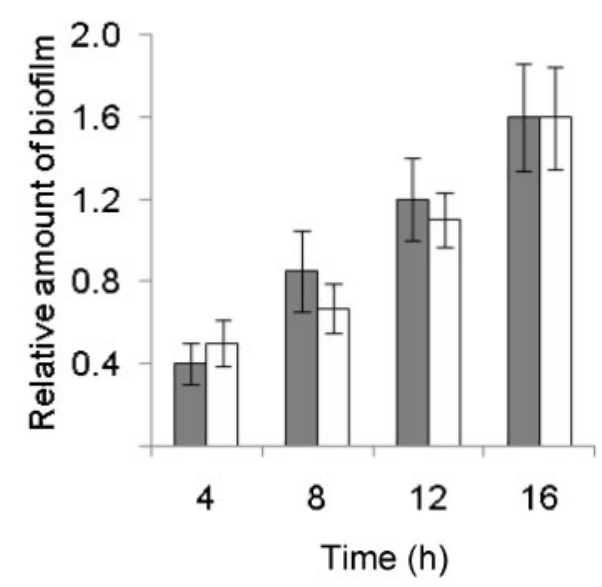

Fig. 3. The $i b p A B$ mutation does not inhibit air-liquid biofilm growth at $37^{\circ} \mathrm{C}$. E. coli WT (grey bars) and ibpAB (white bars) were grown at $37{ }^{\circ} \mathrm{C}$ in PVC microtitre plates as described in the legend to Fig. 1. At the indicated time points the relative amount of biofilm was determined as described in Methods. The data correspond to the means \pm SD of three independent experiments. $i b p A B$ cells. The fact that (1) IbpA and IbpB participate in the protection of $E$. coli cells against oxidative damage (Kitagawa et al., 2000, 2002; Matuszewska et al., 2008) and (2) the TnaA level increases during oxidative stress (Pomposiello et al., 2001; Zheng et al., 2001) prompted the hypothesis that cells lacking IbpAB proteins are exposed to endogenous oxidative stress which leads to the overproduction of TnaA and indole. To investigate this possibility, we first compared the level of oxidation of $\mathrm{H}_{2}$ DCFDA in WT and ibpAB cells. The oxidation of $\mathrm{H}_{2}$ DCFDA depends on the level of reactive oxygen species and reduced glutathione; therefore, it is commonly used to detect general oxidative stress in cells (Jakubowski et al., 2000; Echave et al., 2003). We found that, independently of the phase of growth, the oxidation of $\mathrm{H}_{2}$ DCFDA was enhanced in the $i b p A B$ strain by $30-40 \%$, as compared to WT cells (Fig. 4a), confirming that E. coli $i b p A B$ was exposed to endogenous oxidative stress. Further evidence supporting this hypothesis was provided by immunodetection of oxidized proteins in WT and $i b p A B$ cells. Elevated protein oxidation was observed in $i b p A B$ cells up to $6 \mathrm{~h}$ after inoculation (Fig. 4b). The protein band which appeared in the second hour of culture in the $i b p A B$ cells, but was absent in the WT strain, corresponded to the oxidized EF-G protein, according to the literature (Tamarit et al., 1998). The amount and pattern of carbonylated proteins were not changed significantly after the sixth hour of culture (data not shown). Thus, in the absence of IbpAB 
(a)

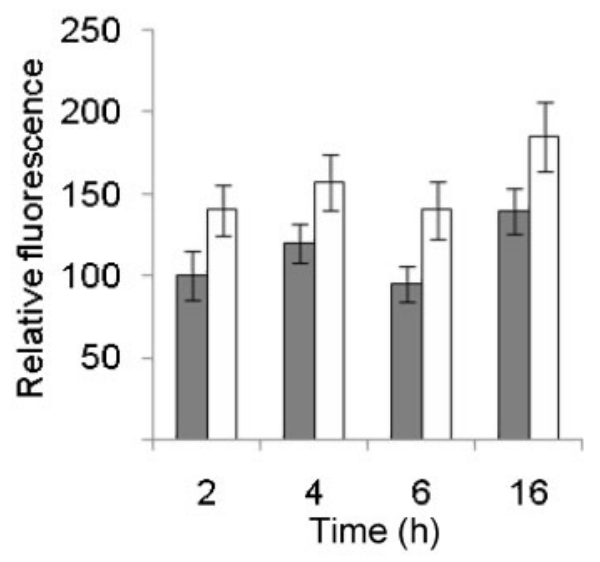

(b)

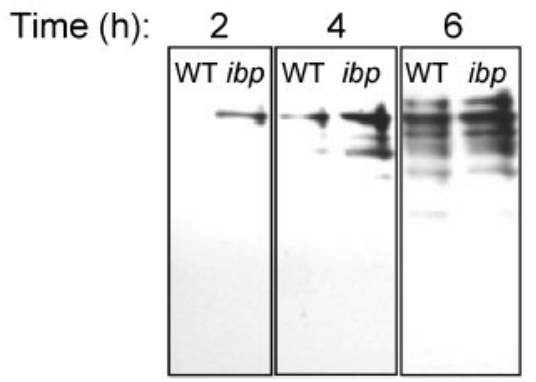

Fig. 4. The $i b p A B$ strain is challenged with endogenous oxidative stress. E. coli WT (grey bars) and ibpAB (white bars) were grown as described in the legend to Fig. 1. (a) At the indicated time points, the oxidation of $\mathrm{H}_{2}$ DCFDA was measured as arbitrary units of fluorescence intensity. The data correspond to the means \pm SD of three independent experiments. The observed difference between WT and ibpAB strains was found to be significant $(P<0.05)$ in a paired two-tailed $t$ test. (b) Protein carbonyl groups were detected in the same samples. After derivatization with DNPH (see Methods), aliquots containing equal amounts of protein were resolved by SDSPAGE and analysed using an anti-DNP antibody.

some E. coli proteins became more sensitive to oxidation during the early exponential phase. However, the damage caused by endogenous oxidative stress did not affect the growth of E. coli ibpAB (Fig. 1a).

Next, we measured two enzymic activities in $\mathrm{WT}$ and $i b p A B$ cells: those of catalase and superoxide dismutase (SOD), which participate in antioxidant defence and neutralize $\mathrm{H}_{2} \mathrm{O}_{2}$ and superoxide ions, respectively. Interestingly, in the $i b p A B$ strain catalase activity was decreased by $25-30 \%$, when compared to WT cells (Fig. 5a), while SOD activity was not affected (Fig. 5b). It should be pointed out that in the complementation tests, catalase activity in the $\operatorname{ibp} A B(\mathrm{pGab})$ culture reached the levels detected in the WT cells. These results suggest that inefficient catalase activity in the $i b p A B$ mutant might be responsible for the elevated endogenous oxidative stress $\left(\mathrm{H}_{2} \mathrm{O}_{2}\right)$ and increased level of oxidized proteins. This, in (a)
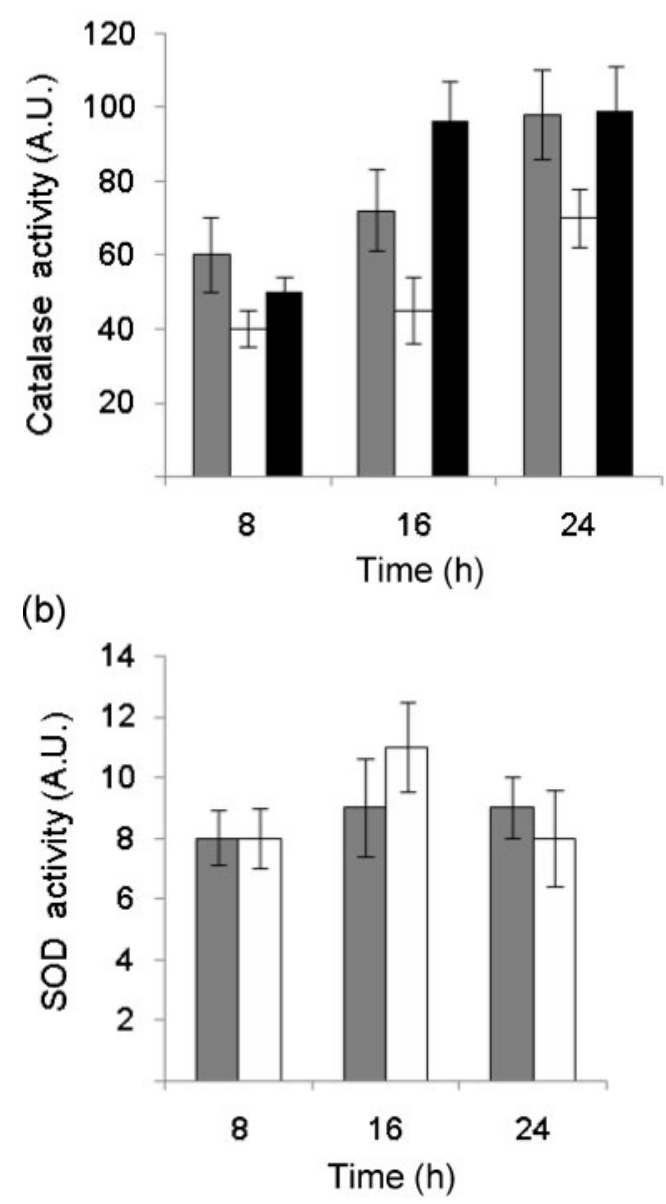

Fig. 5. The $i b p A B$ mutation affects catalase activity in E. coli cells. $E$. coli strains were grown in PVC microtitre plates as described in Methods. (a) Catalase activity was determined in cell extracts obtained from cultures of E. coli WT(pGB2) (grey bars), ibp $A B$ (pGB2) (white bars) and ibpAB(pGab) (black bars), which contained both planktonic and biofilm cells. (b) SOD activity was determined in extracts of WT (grey bars) and $i b p A B$ (white bars). Means $\pm S D$ of three independent experiments are shown. A.U., arbitrary units.

turn, may lead to the overproduction of TnaA and extracellular indole, resulting in biofilm suppression. To verify this hypothesis we tested the ability of catalase mutant strains to form biofilm. E. coli has two catalase enzymes, HPI and HPII (Visick \& Clarke, 1997). HPI, the product of the kat $G$ gene, is induced during exponential growth in response to low concentrations of hydrogen peroxide, and at the stationary phase in a $\sigma^{\mathrm{S}}$-independent way. The katE gene, which codes for HPII, is not peroxideinducible, but is activated at the transition from the exponential to the stationary phase by RNA polymerase containing the $\sigma^{\mathrm{S}}$ subunit. We found that the lack of HPI catalase, but not HPII, resulted in the delay of E. coli biofilm formation at the air-liquid interface (Fig. 6a). 
Moreover, increased TnaA activity (Fig. 6b) and an elevated level of extracellular indole (Fig. 6c) were detected in the mid-exponential-phase $k a t G$ cultures. The enzyme activity and production of extracellular indole were only slightly affected by the katE deletion (Fig. 6b, c). These data indicated that the lack of $\mathrm{IbpAB}$ proteins resulted in diminished HPI activity, leading to the overproduction of extracellular indole and inhibition of biofilm formation.

\section{Fluidization of $E$. coli membranes by benzyl alcohol inhibits biofilm formation}

We found that in the presence of $\mathrm{Cu}(\mathrm{II})$, the inhibitory effect of the $i b p A B$ mutation on biofilm growth was stronger than in non-stressed cultures (compare Fig. 1d and Fig. 1b). It is known that copper stress influences permeability of membranes (Suwalsky et al., 1998). It was also reported that the $i b p A B$ mutation increases membrane fluidity and permeability (Nakamoto \& Vigh, 2007); therefore $\mathrm{Cu}(\mathrm{II})$ can cause more severe damage to the $i b p A B$ cell membranes, leading to further inhibition of biofilm formation. It is possible that the altered physical state of the membranes contributes to the suppression of $i b p A B$ biofilm growth in the absence of $\mathrm{Cu}(\mathrm{II})$ also. Interestingly, we found that nonlethal doses of a membrane fluidizer, benzyl alcohol (Shigapova et al., 2005), increased the level of extracellular indole (although it did not influence TnaA activity) in the WT culture. Simultaneously, benzyl alcohol significantly suppressed biofilm formation (Fig. 7). This result suggests that the fluidization and permeabilization of E. coli membranes caused by the lack of IbpAB proteins (Nakamoto \&Vigh, 2007) may contribute to reduced biofilm formation by $i b p A B$ cells.

\section{DISCUSSION}

E. coli biofilm development is a complex process which requires the coordinated expression of more than 100 genes (reviewed by Prüß et al., 2006; Wood, 2009). Gene expression profiles in E. coli biofilms have identified various stress-response genes as being important during the growth of the biofilm. The stress-response genes overexpressed in biofilms are involved in the SOS response ( $\operatorname{din} I, \operatorname{din} P, \operatorname{din} G, \operatorname{sbm} C, \operatorname{rec} N, \operatorname{sul} A)$, envelope stress ( $p s p A B C D E, c p x A R, r p o E)$, general stress response (rpoS, $y c f R$ ) and heat stress (dnaK, dnaJ, clpB, ibpA, ibpB) (Schembri et al., 2003; Beloin et al., 2004; Ren et al., 2004, Junker et al., 2007; Domka et al., 2007). Compared to the role of the surface proteins directly involved in cell attachment, little is known about the function of molecular chaperones in biofilm development. It was reported that DnaK, DnaJ and GrpE are required for the synthesis of flagella (Shi et al., 1992). DnaK and DnaJ are also involved in the regulation of synthesis of cyclic lipopeptides, putisolvins, which possess surfactant activity and play an important role in the formation of Pseudomonas putida biofilm (Dubern et al., 2005).
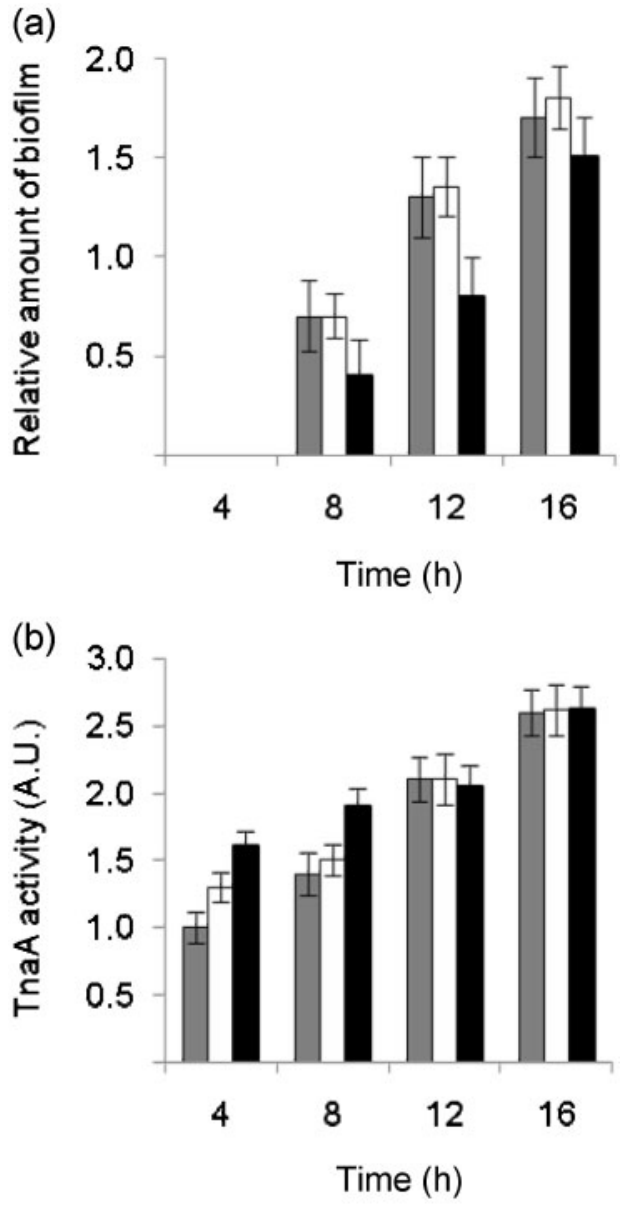

(c)

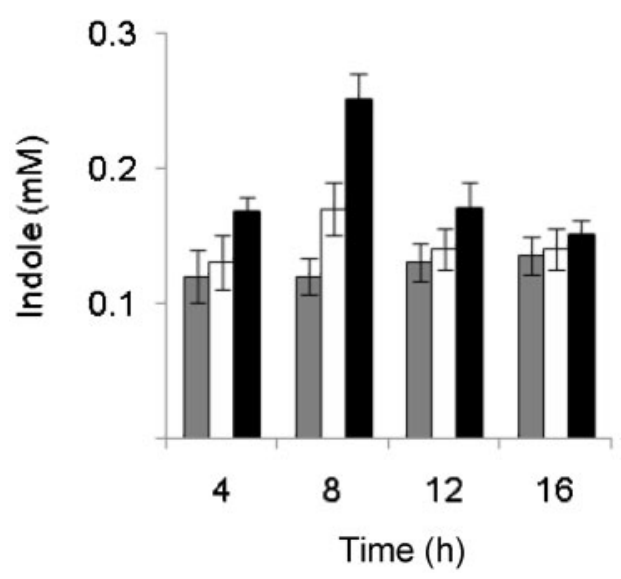

Fig. 6. The katG mutation inhibits formation of $E$. coli biofilm. $E$. coli WT (grey bars), katE (white bars) and katG (black bars) strains were grown in PVC microtitre plates as described in Methods. (a) At the indicated time points, relative amounts of biofilm were estimated. (b) TnaA activity was measured in cell extracts obtained from cultures containing both planktonic and biofilm cells. A.U., arbitrary units. (c) The level of extracellular indole was determined in spent medium collected after the sedimentation of planktonic cells. TnaA activities and indole levels were normalized to cell density. Means \pm SD of three independent experiments are shown. 


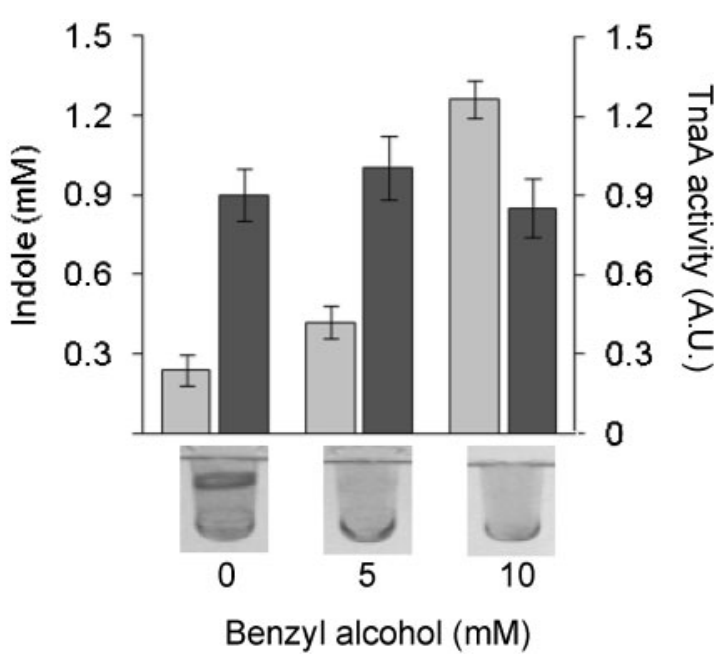

Fig. 7. Benzyl alcohol increases the level of extracellular indole and inhibits biofilm formation. The E. coli WT strain was grown in the presence of increased concentrations of benzyl alcohol in PVC microtitre plates as decribed in Methods. After $3 \mathrm{~h}, \mathrm{TnaA}$ activity (dark grey bars) and extracellular concentrations of indole (light grey bars) were measured. Biofilm was detected by crystal violet staining after $16 \mathrm{~h}$ of incubation (photographs). Means \pm SD of three independent experiments and representative wells are shown. A.U., arbitrary units.

In this study we focused on the role of the IbpAB proteins, which have been previously reported to protect $E$. coli cells against heat and oxidative stresses (Kuczyńska-Wisńik et al., 2002; Matuszewska et al., 2008). The results presented in this paper demonstrate that the lack of IbpAB proteins in E. coli cells allows normal planktonic growth but inhibits the formation of biofilm at the air-liquid interface. We propose that an increased concentration of extracellular indole in the $\triangle i b p A B$ culture is responsible for this inhibitory effect. Recently, it has been proposed that indole plays the role of an extracellular signal (Wang et al., 2001), inhibiting development of E. coli biofilms (Lee et al., 2008). However, other studies in which different E. coli strains and media were used suggested that indole may increase biofilm formation ability (Di Martino et al., 2003; Collet et al., 2007). Intriguingly, indole may also function as an interspecies signal which increases the formation of the biofilms of Pseudomonas aeruginosa and P. fluorescens, bacteria which do not produce this signal (Lee et al., 2007). Indole is a product of the reaction catalysed by tryptophanase, an increased level of which was detected in $i b p A B$ cells (Fig. 2a, b). It is known that tnaA expression can be induced in the presence of reactive oxygen species (Pomposiello et al., 2001; Zheng et al., 2001). We showed that cells lacking the IbpAB proteins are exposed to endogenous oxidative stress, which might lead to enhanced expression of the tnaA gene. Using $\mathrm{H}_{2} \mathrm{DCFDA}$ as a probe, we detected an increased level of general oxidative stress (Fig. 4a) and found elevated amounts of oxidized proteins in $i b p A B$ cells (Fig. 4b). These results indicated that the mutant was indeed exposed to endogenous oxidative stress. Determination of the two main enzyme activities involved in the antioxidant protection (catalase and SOD) revealed that the $i b p A B$ strain also exhibited a decreased catalase activity (Fig. 5). Since it was reported that IbpAB proteins protect various enzymes against oxidative damage (Kitagawa et al., 2002; Matuszewska et al., 2008), it is possible that these proteins also prevent inactivation of catalase(s). The method used in this work for the quantification of catalase activity did not allow us to distinguish which of the two E. coli catalases, HPI (the product of the kat $G$ gene) or HPII (coded by the katE gene), was affected by the $i b p A B$ mutation. Further studies revealed that the lack of HPI, but not HPII, delayed airliquid interface biofilm formation (Fig. 6a), accompanied by increased TnaA activity and overproduction of extracellular indole (Fig. 6b, c) in mid-exponential-phase cultures. A significant increase in the level of extracellular indole in the $k a t G$ culture was observed only at the eighth hour of culture (Fig. 6c), which may indicate that mechanisms were initiated in kat $G$ cells that counteract further overproduction of extracellular indole. Hence, the effect of the kat $G$ mutation on biofilm growth (Fig. 6a) was less evident than in the case of $i b p A B$ cells (Fig. 1b). It should be noted that HPI is produced at the exponential and stationary phase, whereas HPII is activated at the transition from the exponential to the stationary phase (Visick \& Clarke, 1997), i.e. at the late stage of biofilm growth. Therefore, it is not surprising that the lack of HPII did not influence the early steps of biofilm development at mid-exponential phase (Fig. 6a).

The above results suggested that detoxification of intracellular $\mathrm{H}_{2} \mathrm{O}_{2}$ (a by-product of aerobic metabolism) by HPI was impaired in the absence of IbpAB. How the endogenous oxidative stress $\left(\mathrm{H}_{2} \mathrm{O}_{2}\right)$ led to the induction of tnaA and overproduction of extracellular indole in $i b p A B$ cells remains unclear. A similar induction mechanism seems to exist in the WT strain exposed to exogenous $\mathrm{H}_{2} \mathrm{O}_{2}$. We found that WT cells grown in the presence of $5 \mathrm{mM} \mathrm{H}_{2} \mathrm{O}_{2}$ exhibited higher (by $25 \%$ ) TnaA activity and produced twofold more indole compared to non-stressed WT cultures. Furthermore, $\mathrm{H}_{2} \mathrm{O}_{2}$ did not affect growth of the strain, but significantly delayed WT biofilm formation (data not shown). The inhibitory effect of oxidative and heavy metal stresses on biofilm formation was also found in E. coli cultures exposed to paraquat, $\mathrm{Cd}(\mathrm{II})$ (data not shown) and $\mathrm{Cu}$ (II) (Fig. 6). It is important to note that Zhang et al. (2007) observed the opposite effect of oxidative and heavy metal stress on E. coli biofilm. They reported that $\mathrm{H}_{2} \mathrm{O}_{2}$ and $\mathrm{Cd}(\mathrm{II})$ strongly stimulated growth of E. coli BW25113 biofilm at $37^{\circ} \mathrm{C}$, in non-buffered LB-medium. Interestingly, this enhanced biofilm formation was accompanied by decreased indole synthesis. Therefore, the data published by Zhang et al. (2007) and our results demonstrate that oxidative and heavy metal stress may affect (increase or decrease) biofilm 
formation by influencing the extracellular indole concentration. The differences between their studies and ours could result from the use of different E. coli strains and growth conditions.

We demonstrated that the inhibitory effect of the ibpAB mutation on biofilm formation was stronger in the presence of $\mathrm{Cu}(\mathrm{II})$ than in non-treated cultures (compare Fig. 1e and Fig. 1b). The mechanism of copper cytotoxicity is complex and includes the generation of reactive oxygen species and perturbations of membrane permeability (Suwalsky et al., 1998; Kershaw et al., 2005). We hypothesize that membrane damage may be one of the reasons for the inhibition of biofilm formation under $\mathrm{Cu}(\mathrm{II})$-induced stress. Perturbed membrane permeability may impair synthesis, export and maintenance of various cell surface molecules and structures (flagella, type 1 fimbriae, curli and extracellular polymeric substances) implicated in different stages of biofilm formation (Van Houdt \& Michiels, 2005). Moreover, the activity of innermembrane proteins AcrEF and Mtr, responsible for the export and import of indole, respectively (Wood, 2009), could also be affected, leading to an increased concentration of extracellular indole. It is known that $i b p A B$ mutation decreases membrane stability (Nakamoto \& Vigh, 2007); therefore $\mathrm{Cu}(\mathrm{II})$ can cause more severe damage to $i b p A B$ cell membranes, reflected in the additional inhibition of biofilm formation. Our experiment with the membrane fluidizer benzyl alcohol provided further evidence that the altered physical state of membranes contributes to the suppression of biofilm growth. We demonstrated that non-lethal doses of benzyl alcohol increased the extracellular level of indole and significantly suppressed biofilm formation (Fig. 7).

In summary, we have demonstrated that the IbpAB proteins accelerate formation of E. coli biofilm by protecting cells against endogenous oxidative stress. In the absence of IbpAB, E. coli cells experience oxidative stress and overproduce TnaA and extracellular indole. Increased membrane fluidity and permeability in the $i b p A B$ mutant may also contribute to the increased extracellular concentration of indole, which reduces biofilm formation.

\section{ACKNOWLEDGEMENTS}

This work was supported by UG grant BW/ L130-5-0094-9 and grant 0923/B/P01/2009/36 from the Polish Ministry of Science and Higher Education. We are grateful to Professor Barbara Lipińska (University of Gdańsk, Poland) for discussions and critical reading of the manuscript. We thank Professor Jorge Membrillo-Hernández (Instituto de Investigaciones Biomédicas, Mexico City, Mexico) for providing strain W3110 and helpful suggestions regarding biofilm growth.

\section{REFERENCES}

Allen, S. P., Polazzi, J. O., Gierse, J. K. \& Easton, A. M. (1992). Two novel heat shock genes encoding proteins produced in response to heterologous protein expression in Escherichia coli. J Bacteriol 174, 6938-6947.

Beloin, C., Valle, J., Latour-Lambert, P., Faure, P., Kzreminski, M., Balestrino, D., Haagensen, J. A., Molin, S., Prensier, G. \& other authors (2004). Global impact of mature biofilm lifestyle on Escherichia coli K-12 gene expression. Mol Microbiol 51, 659-674.

Blankenhorn, D., Phillips, J. \& Slonczewski, J. L. (1999). Acid- and base-induced proteins during aerobic and anaerobic growth of Escherichia coli revealed by two-dimensional gel electrophoresis. J Bacteriol 181, 2209-2216.

Choi, S. S., Kang, B. Y., Chung, M. J., Kim, S. D., Park, S. H., Kim, J. S., Kang, C. Y. \& Ha, N. J. (2005). Safety assessment of potential lactic acid bacteria Bifidobacterium longum SPM1205 isolated from healthy Koreans. J Microbiol 43, 493-498.

Chuang, S. E., Burland, V., Plunkett, G., III, Daniels, D. L. \& Blattner, F. R. (1993). Sequence analysis of four new heat-shock genes constituting the hslTS/ibpAB and hslVU operons in Escherichia coli. Gene 134, 1-6.

Churchward, G., Belin, D. \& Nagamine, Y. (1984). A pSC101-derived plasmid which shows no sequence homology to other commonly used cloning vectors. Gene 31, 165-171.

Collet, A., Vilain, S., Cosette, P., Junter, G. A., Jouenne, T., Phillips, R. S. \& Di Martino, P. (2007). Protein expression in Escherichia coli S17-1 biofilms: impact of indole. Antonie Van Leeuwenhoek 91, 71-85.

Colón-González, M., Méndez-Ortiz, M. M. \& Membrillo-Hernández, J. (2004). Anaerobic growth does not support biofilm formation in Escherichia coli K-12. Res Microbiol 155, 514-521.

Di Martino, P., Fursy, R., Bret, L., Sundararaju, B. \& Phillips, R. S. (2003). Indole can act as an extracellular signal to regulate biofilm formation of Escherichia coli and other indole-producing bacteria. Can J Microbiol 49, 443-449.

Domka, J., Lee, J. \& Wood, T. K. (2006). YliH (BssR) and YceP (BssS) regulate Escherichia coli K-12 biofilm formation by influencing cell signaling. Appl Environ Microbiol 72, 2449-2459.

Domka, J., Lee, J., Bansal, T. \& Wood, T. K. (2007). Temporal geneexpression in Escherichia coli K-12 biofilms. Environ Microbiol 9, 332346.

Dubern, J. F., Lagendijk, E. L., Lugtenberg, B. J. \& Bloemberg, G. V. (2005). The heat shock genes $d n a K, d n a J$, and grpE are involved in regulation of putisolvin biosynthesis in Pseudomonas putida PCL1445. J Bacteriol 187, 5967-5976.

Echave, P., Tamarit, J., Cabiscol, E. \& Ros, J. (2003). Novel antioxidant role of alcohol dehydrogenase E from Escherichia coli. J Biol Chem 278, 30193-30198.

Fux, C. A., Costerton, J. W., Stewart, P. S. \& Stoodley, P. (2005). Survival strategies of infectious biofilms. Trends Microbiol 13, 34-40.

Hall-Stoodley, L., Costerton, J. W. \& Stoodley, P. (2004). Bacterial biofilms: from the natural environment to infectious diseases. Nat Rev Microbiol 2, 95-108.

Harrison, J. J., Ceri, H. \& Turner, R. J. (2007). Multimetal resistance and tolerance in microbial biofilms. Nat Rev Microbiol 5, 928-938.

Haslbeck, M., Franzmann, T., Weinfurtner, D. \& Buchner, J. (2005). Some like it hot: the structure and function of small heat-shock proteins. Nat Struct Mol Biol 12, 842-846.

Jakubowski, W., Biliński, T. \& Bartosz, G. (2000). Oxidative stress during aging of stationary cultures of the yeast Saccharomyces cerevisiae. Free Radic Biol Med 28, 659-664.

Junker, L. M., Toba, F. A. \& Hay, A. G. (2007). Transcription in Escherichia coli PHL628 biofilms. FEMS Microbiol Lett 268, 237-243.

Kershaw, C. J., Brown, N. L., Constantinidou, C., Patel, M. D. \& Hobman, J. L. (2005). The expression profile of Escherichia coli K-12 
in response to minimal, optimal and excess copper concentrations. Microbiology 151, 1187-1198.

Kitagawa, M., Miyakawa, M., Matsumura, Y. \& Tsuchido, T. (2000). Small heat shock proteins, IbpA and IbpB, are involved in resistances to heat and superoxide stress in Escherichia coli. FEMS Microbiol Lett 184, 165-171.

Kitagawa, M., Miyakawa, M., Matsumura, Y. \& Tsuchido, T. (2002). Escherichia coli small heat shock proteins, IbpA and IbpB, protect enzymes from inactivation by heat and oxidants. Eur J Biochem 269, 2907-2917.

Kuczyńska-Wisńik, D., Laskowska, E. \& Taylor, A. (2001). Transcription of the $i b p B$ heat-shock gene is under control of $\sigma^{32}$ and $\sigma^{54}$-promoters, a third regulon of heat-shock response. Biochem Biophys Res Commun 284, 57-64.

Kuczyńska-Wisńik, D., Kędzierska, S., Matuszewska, E., Lund, P., Taylor, A., Lipińska, B. \& Laskowska, E. (2002). The Escherichia coli small heat-shock proteins IbpA and IbpB prevent the aggregation of endogenous proteins denatured in vivo during extreme heat shock. Microbiology 148, 1757-1765.

Lacour, S. \& Landini, P. (2004). $\sigma^{\mathrm{S}}$-dependent gene expression at the onset of stationary phase in Escherichia coli: function of $\sigma^{\mathrm{S}}$-dependent genes and identification of their promoter sequences. J Bacteriol 186, 7186-7195.

Laskowska, E., Wawrzynów, A. \& Taylor, A. (1996). IbpA and IbpB, the new heat shock proteins, bind to endogenous Escherichia coli proteins aggregated intracellularly by heat shock. Biochimie 78, 117122.

Laskowska, E., Kuczyńska-Wiśnik, D., Bąk, M. \& Lipińska, B. (2003). Trimethoprim induces heat shock proteins and protein aggregation in E. coli cells. Curr Microbiol 47, 286-289.

Lee, J., Jayaraman, A. \& Wood, T. K. (2007). Indole is an inter-species biofilm signal mediated by SdiA. BMC Microbiol 7, 42.

Lee, J., Zhang, X. S., Hegde, M., Bentley, W. E., Jayaraman, A. \& Wood, T. K. (2008). Indole cell signaling occurs primarily at low temperatures in Escherichia coli. ISME J 2, 1007-1023.

Matuszewska, M., Kuczyńska-Wiśnik, D., Laskowska, E. \& Liberek, K. (2005). The small heat shock protein IbpA from Escherichia coli cooperates with $\mathrm{IbpB}$ in stabilization of thermally aggregated proteins in a disaggregation competent state. J Biol Chem 280, 12292-12298.

Matuszewska, E., Kwiatkowska, J., Kuczyńska-Wiśnik, D. \& Laskowska, E. (2008). Escherichia coli heat-shock proteins IbpA/B are involved in resistance to oxidative stress induced by copper. Microbiology 154, 1739-1747.

Mogk, A., Deuerling, E., Vorderwülbecke, S., Vierling, E. \& Bukau, B. (2003a). Small heat shock proteins, ClpB and the DnaK system form a functional triade in reversing protein aggregation. Mol Microbiol 50, 585-595.

Mogk, A., Schlieker, C., Friedrich, K. L., Schönfeld, H. J., Vierling, E. \& Bukau, B. (2003b). Refolding of substrates bound to small Hsps relies on a disaggregation reaction mediated most efficiently by ClpB/DnaK. J Biol Chem 278, 31033-31042.

Nakamoto, H. \& Vigh, L. (2007). The small heat shock proteins and their clients. Cell Mol Life Sci 64, 294-306.

Nobre, L. S., Al-Shahrour, F., Dopazo, J. \& Saraiva, L. M. (2009). Exploring the antimicrobial action of a carbon monoxide-releasing compound through whole-genome transcription profiling of Escherichia coli. Microbiology 155, 813-824.

Peréz, J. M., Calderón, I. L., Arenas, F. A., Fuentes, D. E., Pradenas, G. A., Fuentes, E. L., Sandoval, J. M., Castro, M. E., Elías, A. O. \&
Vásquez, C. C. (2007). Bacterial toxicity of potassium tellurite: unveiling an ancient enigma. PLoS One 2, e211.

Pomposiello, P. J., Bennik, M. H. \& Demple, B. (2001). Genome-wide transcriptional profiling of the Escherichia coli responses to superoxide stress and sodium salicylate. J Bacteriol 183, 3890-3902.

PrüB, B. M., Besemann, C., Denton, A. \& Wolfe, A. J. (2006). A complex transcription network controls the early stages of biofilm development by Escherichia coli. J Bacteriol 188, 3731-3739.

Ratajczak, E., Zietkiewicz, S. \& Liberek, K. (2009). Distinct activities of Escherichia coli small heat shock proteins IbpA and IbpB promote efficient protein disaggregation. J Mol Biol 386, 178-189.

Ren, D., Bedzyk, L. A., Thomas, S. M., Ye, R. W. \& Wood, T. K. (2004). Gene expression in Escherichia coli biofilms. Appl Microbiol Biotechnol 64, 515-524.

Sambrook, J., Fritsh, E. F. \& Maniatis, F. (1989). Molecular Cloning: a Laboratory Manual, 2nd edn.Cold Spring Harbor, NY: Cold Spring Harbor Laboratory.

Schembri, M. A., Kjaergaard, K. \& Klemm, P. (2003). Global gene expression in Escherichia coli biofilms. Mol Microbiol 48, 253-267.

Shi, W., Zhou, Y., Wild, J., Adler, J. \& Gross, C. A. (1992). DnaK, DnaJ, and GrpE are required for flagellum synthesis in Escherichia coli. J Bacteriol 174, 6256-6263.

Shigapova, N., Török, Z., Balogh, G., Goloubinoff, P., Vígh, L. \& Horváth, I. (2005). Membrane fluidization triggers membrane remodeling which affects the thermotolerance in Escherichia coli. Biochem Biophys Res Commun 328, 1216-1223.

Stewart, P. S. \& Franklin, M. J. (2008). Physiological heterogeneity in biofilms. Nat Rev Microbiol 6, 199-210.

Suwalsky, M., Ungerer, B., Quevedo, L., Aguilar, F. \& Sotomayor, C. P. (1998). $\mathrm{Cu}^{2+}$ ions interact with cell membranes. J Inorg Biochem 70, 233-238.

Tamarit, J., Cabiscol, E. \& Ros, J. (1998). Identification of the major oxidatively damaged proteins in Escherichia coli cells exposed to oxidative stress. J Biol Chem 273, 3027-3032.

Van Houdt, R. \& Michiels, C. W. (2005). Role of bacterial cell surface structures in Escherichia coli biofilm formation. Res Microbiol 156, 626-633.

Veinger, L., Diamant, S., Buchner, J. \& Goloubinoff, P. (1998). The small heat-shock protein IbpB from Escherichia coli stabilizes stress denatured proteins for subsequent refolding by a multichaperone network. J Biol Chem 273, 11032-11037.

Visick, J. E. \& Clarke, S. (1997). RpoS- and OxyR-independent induction of HPI catalase at stationary phase in Escherichia coli and identification of rpoS mutations in common laboratory strains. J Bacteriol 179, 4158-4163.

Wang, D., Ding, X. \& Rather, P. N. (2001). Indole can act as an extracellular signal in Escherichia coli. J Bacteriol 183, 4210-4216.

Wood, T. K. (2009). Insights on Escherichia coli biofilm formation and inhibition from whole-transcriptome profiling. Environ Microbiol 11, $1-15$.

Zhang, X. S., Garcia-Contreras, R. \& Wood, T. K. (2007). YcfR (BhsA) influences Escherichia coli biofilm formation through stress response and surface hydrophobicity. J Bacteriol 189, 3051-3062.

Zheng, M., Wang, X., Templeton, L. J., Smulski, D. R., La Rossa, R. A. \& Storz, G. (2001). DNA microarray-mediated transcriptional profiling of the Escherichia coli response to hydrogen peroxide. J Bacteriol 183, 4562-4570.

Edited by: J.-H. Roe 